\title{
Pure-Pursuit Reactive Path Tracking for Nonholonomic Mobile Robots with a 2D Laser Scanner
}

\author{
Jesús Morales, Jorge L. Martínez, María A. Martínez, and Anthony Mandow \\ E.T.S. Ingenieros Industriales, Universidad de Málaga, 29071 Málaga, Spain \\ Correspondence should be addressed to Jesús Morales, jesus.morales@uma.es
}

Received 31 July 2008; Revised 5 December 2008; Accepted 30 January 2009

Recommended by Matthijs Spaan

\begin{abstract}
Due to its simplicity and efficiency, the pure-pursuit path tracking method has been widely employed for planned navigation of nonholonomic ground vehicles. In this paper, we investigate the application of this technique for reactive tracking of paths that are implicitly defined by perceived environmental features. Goal points are obtained through an efficient interpretation of range data from an onboard 2D laser scanner to follow persons, corridors, and walls. Moreover, this formulation allows that a robotic mission can be composed of a combination of different types of path segments. These techniques have been successfully tested in the tracked mobile robot Auriga- $\alpha$ in an indoor environment.
\end{abstract}

Copyright (C) 2009 Jesús Morales et al. This is an open access article distributed under the Creative Commons Attribution License, which permits unrestricted use, distribution, and reproduction in any medium, provided the original work is properly cited.

\section{Introduction}

A mobile robot path can be defined as the course that the vehicle has to follow in the environment. It should be useful for carrying out the mobile robot mission, and admissible from the vehicle's kinematic and dynamic standpoints.

Path following is performed by the path tracking controller. Its goal is to autonomously drive the mobile robot along the path by continually generating speed and steering commands that compensate for the tracking errors. These mainly consist of vehicle's deviations in distance and heading from the path. Both feedback and feedforward control mechanisms are employed for this purpose, with a tradeoff between control effort and control error.

Some path tracking methods for ground vehicles are based on nonlinear control theory, such as PredictiveControl [1] or Fuzzy-Control [2]. Alternatively, geometric considerations between the current vehicle pose and the path to follow can provide simpler tracking strategies [3].

A widespread and effective geometric method is the purepursuit algorithm [3]. It calculates a circumference arc that joins the current position of the vehicle and a goal point in the path. This point is chosen at a specified lookahead distance, which is the chord length of this arc. The advantages of this method include the ease of tuning of the lookahead distance, its computational simplicity, and the absence of derivative terms.

Paths for mobile robots can be classified broadly as explicit or implicit. An explicit path is defined in a global frame as a sequence of way-point coordinates that are joined by straight line segments [4] or by adjusting a parametric curve [5]. In this case, tracking error computation involves real-time estimation of the pose of the vehicle with respect to the path. Usually, this implies processing signals from various sensors (e.g., GPS, odometry, range, or vision) and relating them with a geometric model of the environment [6].

On the other hand, an implicit path is described by remarkable characteristics that are perceivable in the environment with an appropriate set of sensors. For example, a road determines a path that can be recognized in an image by its left and right edges [7], and a person course can be detected as point clusters in consecutive range scans [8]. Thus, computation of the tracking error with respect to an implicit path does not require global pose estimation. This approach allows for a reactive processing if path features can be detected straightforwardly from sensor data.

The underlying idea of reactive navigation is that only the essential information for a particular path needs to be extracted from sensor data [9]. In this way, it is possible to simplify processing complexity to a great extent. However, 
some problems have to be coped with to implement reactivity. First, dependence on sensor data can lead to a shaky response. Secondly, nonholonomic constraints limit the possible movements from a given pose. Thirdly, current mobile robot sensors can provide a large amount of information that needs to be timely processed.

Many ad hoc approaches and sensor configurations have been devised that develop basic robot control modules to implement high-level functionalities. For example, a pair of ultrasonic sensors allows estimating distance and orientation for wall-following control $[10,11]$. A heterogeneous suite of ultrasonic range sensors and rule-based control was proposed in [12] for following greenhouse aisles. A frequency analysis of sonar echoes is performed in [13] to detect pathway edges based on texture. Some purely reactive strategies have benefited from the accuracy and reliability of laser scanners by using a simplified range ring obtained by discarding most scan angles [14]. Alternatively, the Hough transform of 2D laser scans was proposed in [15] to estimate lateral offset and heading with respect to tree lines. On the other hand, following the course of a moving object (a person or another robot) involves target detection [16] as well as a motion controller for the vehicle to maintain a certain distance to it. In this context, the simplest strategy is to control speed while driving straight in the direction of the current target position. This has been achieved with a camera [17] and with a laser scanner [18]. The fuzzy controller proposed by [19] also considers the variation of the target pose to follow another mobile robot with infrared sensors.

In this paper, we propose a generally applicable approach for reactive motion control that is based on an efficient path tracking method, namely, pure-pursuit, and widely used range-finder devices. This strategy guaranties steering admissibility for nonholonomic vehicles. Also, the lookahead distance can provide a smooth vehicle response. Furthermore, this work proposes a simple interpretation of $2 \mathrm{D}$ range data to reactively compute the goal points.

An additional advantage of our approach is the possibility of employing the basic pure-pursuit strategy as a common frame to follow any combination of implicit and/or explicit paths, which could be efficient in large-scale environments [11]. Particularly, we investigate the application of reactive path tracking to follow persons, walls, and corridors. This has been tested with a tracked mobile robot equipped with a $180^{\circ}$ partial field of view laser scanner in an indoor environment. This kind of sensor has become popular for many field mobile robots due to its small size, reliability, and fast acquisition time [20].

The paper is organized as follows. Section 2 reviews the use of the pure-pursuit method in technical literature. In Section 3, the pure-pursuit strategy is geometrically derived, and its implementation for tracking both explicit and implicit courses is detailed. Section 4 proposes the application of pure-pursuit to follow walls, corridors, and persons autonomously using an onboard 2D laser scanner. In Section 5, experimental results carried out with the mobile robot Auriga- $\alpha$ are presented. Finally, the paper includes conclusions and references.

\section{Literature Review of Pure-Pursuit}

The concept of pure pursuit can be traced in early technical literature regarding the problem of a missile pursuing a target [21]. In the pure pursuit course, the missile velocity vector is always directed toward the instantaneous target position.

Afterwards, in 1985, the pure-pursuit strategy first appears in the field of robotics when Wallace et al. [7] devised a method for calculating the steering necessary to maintain the vehicle on the road. This was accomplished by keeping the road centered in the image obtained from an onboard camera.

Based on this idea, Amidi [3] proposes the pure-pursuit method to follow explicit paths. The name pure-pursuit comes from thinking of the vehicle as chasing a point on the path some distance ahead of it. Some implementation issues of the pure-pursuit algorithm are discussed in the work of Coulter [22]. Since then, the pure-pursuit strategy has been used in many applications for explicit path tracking, including indoor [23] and outdoor navigation [4].

The stability conditions of the pure-pursuit algorithm have been addressed. In this context, the effects of time delays associated with the visual processing for following straightline roads were studied by Murphy [24]. Moreover, stability for tracking explicit paths with constant curvature has been analyzed taking into account computing, communication, and actuators delays in the control loop [25].

The supervision of pure-pursuit parameters was introduced by Ollero et al. [23] as a real-time fuzzy controller that automatically tunes the lookahead distance based on path characteristics, velocity, and tracking errors. A fuzzy-supervised pure-pursuit controller for driving large unmanned vehicles at high speed (above $80 \mathrm{Km} / \mathrm{h}$ ) along explicit paths using differential GPS data was presented by Rodríguez-Castaño et al. [26]. Martínez et al. [27] propose the application of curvature limitations to the pure-pursuit path tracker to avoid interunit collisions in a vehicle that pulls multiple passive trailers.

Due to its widespread use, pure-pursuit has been considered a reference for other path tracking strategies. In [28], it is compared with the follow-the-past algorithm that uses the steering angle and the curvature of the previously recorded explicit path. Regarding person-following with a 2D laser scanner, Gockley et al. [18] present a comparison between a reactive potential-field method and pure-pursuit applied to recorded person positions.

Furthermore, some improvements of the pure-pursuit algorithm have been proposed. In [29], the problem of the vehicle being far away from the path is solved by creating a virtual goal point at the lookahead distance. In [30], the basic pure-pursuit tracker is augmented with an integral correction to reduce systematic tracking errors due to discrepancies between desired and actual steering angles. Stentz et al. [31] add a term proportional to the heading error between the vehicle and the path. Vector pursuit employs the goal point orientation in addition to its position based on screw theory [32].

To sum up, pure-pursuit is a widespread technique that has been analyzed by many authors. However, tracking of 


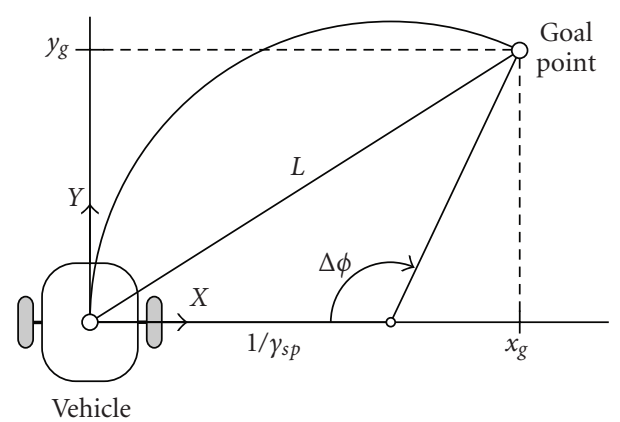

FIGURE 1: The pure-pursuit strategy.

implicit paths has received little attention since the method was first proposed for mobile robots [7], with the exception of our earlier efforts on person following with a rotary sonar [33] and a 2D laser scanner [8]. Moreover, no previous work has explored the possibilities of the method to combine different types of implicit and explicit paths.

\section{The Pure-Pursuit Path Tracker}

To derive the equations of the pure-pursuit curvature control law, let us consider a mobile robot on the plane. The curvature $\gamma$ of the vehicle can be defined as the inverse of the distance $r$ between the vehicle's frame origin and its instantaneous center of rotation (ICR) with sign, that is, the inverse of the turning radius with sign. It is negative if the vehicle turns clockwise and positive otherwise. Curvature also represents the instantaneous change of orientation of the vehicle $d \phi$ with respect to the traveled distance $d s$ as follows:

$$
\gamma=\frac{1}{r}=\frac{d \phi}{d s} .
$$

Let $X Y$ be a right-handed coordinate system attached to the vehicle, whose $Y$ axis is aligned with the forward motion direction (see Figure 1). The local frame origin is chosen so that the local $y$ coordinate of the ICR is always zero, and the curvature is symmetrical with respect to the $Y$ axis.

In the pure-pursuit strategy, the vehicle changes its curvature set-point $\gamma_{s p}$ every control interval $T_{o}$ by fitting a circumference arc to a goal point in the path at a certain lookahead distance $L$. This circumference is tangent to the $Y$ axis of the vehicle (see Figure 1).

Thus, the goal point local coordinates $\left(x_{g}, y_{g}\right)$ can be geometrically obtained as

$$
\begin{aligned}
& x_{g}=\frac{\cos (\Delta \phi)-1}{\gamma_{s p}}, \\
& y_{g}=\frac{\sin (\Delta \phi)}{\gamma_{s p}},
\end{aligned}
$$

where $\Delta \phi$ is the heading change of the vehicle along the arc [3].

Then, by using (2),

$$
L^{2}=x_{g}^{2}+y_{g}^{2}=\frac{2-2 \cos (\Delta \phi)}{\gamma_{s p}^{2}}=-\frac{2 x_{g}}{\gamma_{s p}} .
$$

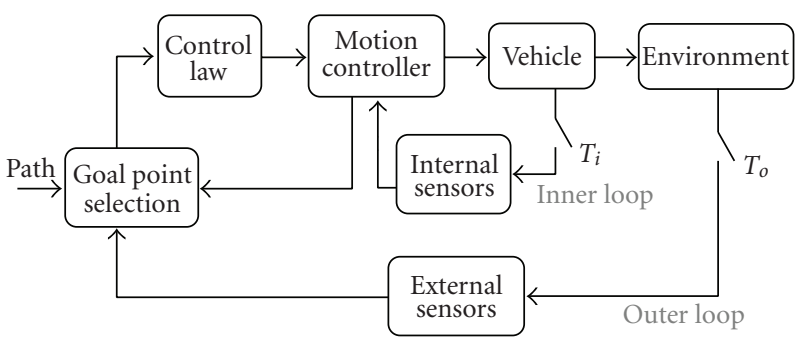

Figure 2: Block diagram of the pure-pursuit path tracker.

Therefore, the curvature control law is given by

$$
\gamma_{s p}=-\frac{2 x_{g}}{L^{2}}
$$

where the terms $-x_{g}$ and $2 / L^{2}$ can be interpreted as the error signal and the gain of the control law, respectively.

The reason for the lookahead distance is that nonholonomic vehicles cannot correct errors with respect to the nearest point of the path. A longer lookahead distance implies a smaller gain, so steering control is smoother at the expense of a worse tracking accuracy. On the other hand, a shorter lookahead can reduce tracking errors, but steering commands increase and the vehicle's motion can become unstable [23].

The pure-pursuit path tracker can be implemented as a cascade controller, as illustrated in Figure 2. The inner loop is a low-level motion controller. It translates speed and steering set-points into references for the actuators, which depend on the locomotion system of the vehicle. The motion controller filters abrupt speed set-point changes according to a maximum acceleration. In some locomotion systems (e.g., differential drive), steering is not completely decoupled from speed. In these cases, speed references $v_{s p}$ are subordinated to curvature set-points $\gamma_{s p}$. The motion controller also updates dead-reckoning with a period $T_{i}$.

The outer loop has a longer control period $T_{o}$. It implements the pure-pursuit control law to issue curvature and speed set-points, which are usually decoupled for simplicity. This control system can be used both for implicit and explicit paths. They differ only in the way that the goal point coordinates are obtained. For explicit paths, external sensors and dead reckoning are needed to localize precisely the vehicle with respect to the path points within the global frame. Otherwise, external sensors are directly employed to estimate the pose of the vehicle with respect to a given type of implicit path in the environment. Then, the curvature setpoint is computed from (4), and the speed set-point can be given by the path.

\section{Implicit Path Tracking with a 2D Laser Scanner}

This section proposes pure-pursuit for reactive tracking of implicit paths with a nonholonomic vehicle. Particularly, this technique has been developed to follow walls, corridors, and persons, based on an onboard 2D laser scanner. For each 


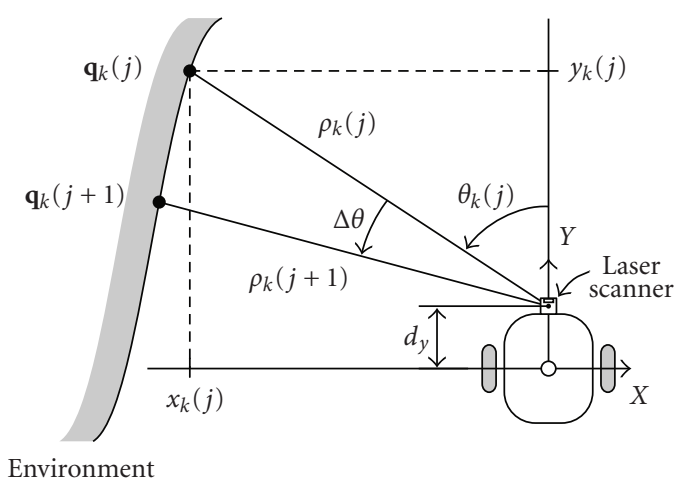

Figure 3: Local cartesian coordinates of the laser point $\mathbf{q}_{k}(j)$.

case, a simple processing of scan data is proposed to achieve a fast computation of the goal point.

A laser scanner mounted on a vehicle generates a light beam that rotates in a horizontal plane parallel to the ground. Thus, a scan at discrete time $k T_{o}$ can be defined as an ordered set of points $\left\{\mathbf{q}_{k}\right\}$. This set corresponds to the successive intersections of the laser ray with the closest objects in the surroundings (see Figure 3 ). The scan is obtained as a list of ranges $\rho_{k}(j)$, where index $j$ is an integer between 0 and $N$. The readings are evenly spaced within the sensor field of view $\Theta$. Then, the beam bearing for $\rho_{k}(j)$ is

$$
\theta_{k}(j)=j \Delta \theta-\frac{\Theta}{2},
$$

where $\Delta \theta=\Theta / N$ represents the angular resolution. The case where $\Theta=360^{\circ}$ corresponds to a complete (or circular) scan sensor. However, partial scan devices with $\Theta=180^{\circ}$ (i.e., semicircular) are commonly employed in mobile robotics.

Note that the laser range-finder position may not coincide with the origin of the vehicle's local frame, even though the central beam direction, that is, $\theta_{k}(N / 2)=0^{\circ}$, is usually made to coincide with its $Y$ axis. This is illustrated in Figure 3, where the laser unit is some distance $d_{y}$ ahead of the local frame. In this case, the local cartesian coordinates for $\mathbf{q}_{k}(j)$ can be computed as follows:

$$
\begin{aligned}
& x_{k}(j)=-\rho_{k}(j) \sin \left(\theta_{k}(j)\right), \\
& y_{k}(j)=d_{y}+\rho_{k}(j) \cos \left(\theta_{k}(j)\right) .
\end{aligned}
$$

4.1. Wall Following. The implicit path for wall following consists of a line that is parallel to the wall at a fixed distance $d_{w}$ (see Figure 4). The goal point should be placed on this line at the distance $L$ ahead of the vehicle's local frame.

Laser data processing consists of finding the shortest range $\rho_{k}^{s}$ and its associated bearing $\theta_{k}^{s}$. This search is limited to positive or negative scan angles depending on whether the wall is at the left or at the right of the vehicle, respectively. Then, the implicit path at time $k T_{o}$ is estimated to be perpendicular to the shortest range beam.

Let us assume an auxiliary right-handed frame $\widehat{X Y}$ whose origin coincides with the local frame. Its $\hat{Y}$ axis is perpendicular to the shortest range direction, and it points

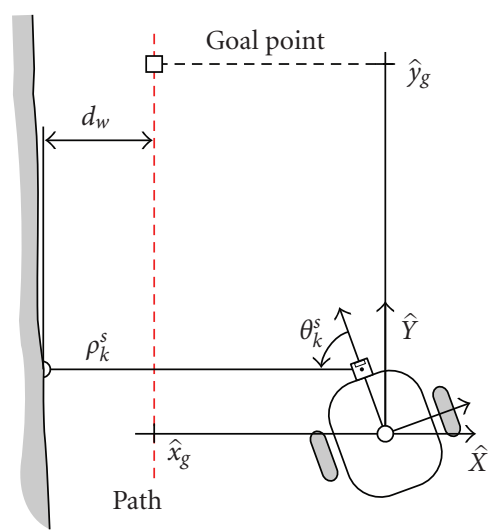

FIGURE 4: Implicit path estimation and goal point selection for left wall following.

forward (see Figure 4). The estimated implicit path is parallel to $\hat{Y}$ so, given $\rho_{k}^{s}$ and $\theta_{k}^{s}$, it can be defined by its coordinate in the $\hat{X}$ axis:

$$
\hat{x}_{p}=\operatorname{sign}\left(\theta_{k}^{s}\right)\left(d_{w}-\rho_{k}^{s}-d_{y} \cos \left(\theta_{k}^{s}\right)\right) .
$$

If $\left|\hat{x}_{p}\right|<L$, then the coordinates of the goal point in the auxiliary frame are

$$
\begin{aligned}
& \hat{x}_{g}=\hat{x}_{p}, \\
& \hat{y}_{g}=\sqrt{L^{2}-\left(\hat{x}_{p}\right)^{2}} .
\end{aligned}
$$

Otherwise, the goal point cannot belong to the implicit path at the lookahead distance $L$. Then, it is computed as follows [29]:

$$
\begin{aligned}
& \hat{x}_{g}=L \operatorname{sign}\left(\hat{x}_{p}\right), \\
& \hat{y}_{g}=0 .
\end{aligned}
$$

Finally, these coordinates need to be rotated to the vehicle's local frame as follows:

$$
x_{g}=\operatorname{sign}\left(\theta_{k}^{s}\right)\left(\sin \left(\theta_{k}^{s}\right) \cos \left(\theta_{k}^{s}\right)\right)\left(\begin{array}{c}
\hat{x}_{g} \\
\hat{y}_{g}
\end{array}\right),
$$

and the curvature set-point is obtained by substituting $x_{g}$ in (4). The speed set-point is a constant value that is specified as a design parameter for the path, which could be chosen depending on $d_{w}$.

The estimation of the implicit path can be inaccurate if a partial scan sensor is used. Whenever the shortest distance to the wall is out of the field of view, the shortest available range corresponds to $\theta_{k}^{s}= \pm \Theta / 2$ (sign depends on wall side). This happens when the vehicle is moving away from the wall and results in a goal point that will be farther from the wall than $d_{w}$. This is illustrated in Figure 5 for a $180^{\circ}$ field of view laser scanner, which produces $\theta_{k}^{s}=90^{\circ}$. Nevertheless, the pure-pursuit method provides an appropriate robot behavior based on this estimation, since the vehicle will rapidly move away from the wall if it is situated at a distance smaller than 


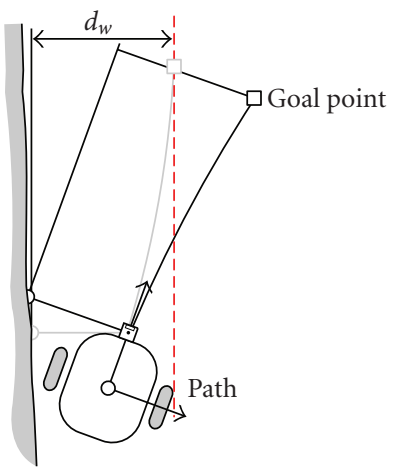

(a)

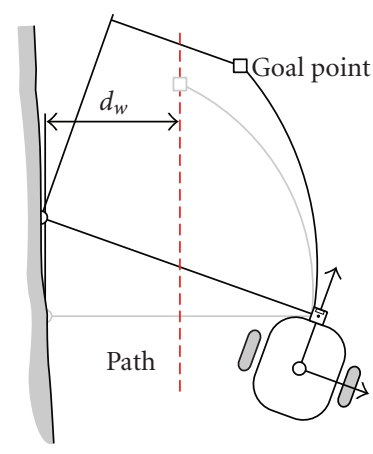

(b)
FIGURE 5: False minimum range with a $180^{\circ}$ partial field of view laser scanner with left wall following, when the robot distance is (a) smaller than $d_{w}$, or (b) greater than $d_{w}$.

$d_{w}$, or it will smoothly approach the implicit path, otherwise, as illustrated in Figures 5(a) and 5(b), respectively.

Real-world walls may include salient features. In this case, the shortest range strategy produces path estimations that momentarily move away from the nominal wall parallel. This way, obstacle collision avoidance is performed by the reactive path tracker.

4.2. Corridor Following. The implicit path is defined by the corridor's midline, and the goal point is chosen on this line at the lookahead distance $L$ of the vehicle.

Let $\widehat{X Y}$ be an auxiliary right-handed frame whose origin coincides with the local frame. The $\hat{Y}$ axis is defined to be parallel to the estimated corridor's midline (see Figure 6). To compute the orientation $\theta^{c}$ of this line with respect to the $Y$ local axis, the shortest ranges at the left $\rho_{k}^{l}$ and at the right $\rho_{k}^{r}$, together with their associated bearings $\theta_{k}^{l}$ and $\theta_{k}^{r}$, are considered. These values are searched within a restricted angle span $\Delta \theta_{\text {th }}$ around $\theta=90^{\circ}$ and $\theta=-90^{\circ}$, respectively. This is necessary to avoid that both selections correspond to the same side of the corridor, especially in L-shaped corridors or with a considerable initial heading error.

For a complete scan sensor, the $x$-coordinates of the left and right sides of the corridor in the auxiliary frame are

$$
\begin{gathered}
\hat{x}^{l}=-\rho_{k}^{l}-d_{y} \cos \left(\theta_{k}^{l}\right), \\
\hat{x}^{r}=\rho_{k}^{r}+d_{y} \cos \left(\theta_{k}^{r}\right),
\end{gathered}
$$

respectively. The orientation angle of the vehicle $\theta^{c}$ with respect to the estimated corridor midline is given by

$$
\theta^{c}=\theta_{k}^{l}+\theta_{k}^{r}
$$

In the case of $180^{\circ}$ partial scan devices, one of the values $\rho_{k}^{l}$ or $\rho_{k}^{r}$ does not actually provide the minimum distance to the corresponding side. In this case,

$$
\theta^{c}=180^{\circ}-\theta_{k}^{l}+\theta_{k}^{r} .
$$

Moreover, if $\theta^{c}>0$ (see Figure 6(a)), then

$$
\hat{x}^{r}=\rho_{k}^{r} \cos \left(\theta^{c}\right)-d_{y} \cos \left(\theta_{k}^{l}\right),
$$

and $\hat{x}^{l}$ is obtained with (11) On the contrary, if $\theta^{c}<0$ (see Figure 6(b)), then

$$
\hat{x}^{l}=-\rho_{k}^{l} \cos \left(\theta^{c}\right)+d_{y} \cos \left(\theta_{k}^{r}\right),
$$

and (12) applies.

In any case, the estimated path line is defined by its $\hat{X}$ coordinate:

$$
\hat{x}_{p}=\frac{\hat{x}^{l}+\hat{x}^{r}}{2}
$$

Then, if $\left|\hat{x}_{p}\right|<L$, the goal point coordinates in the $\widehat{X Y}$ frame are

$$
\begin{aligned}
& \hat{x}_{g}=\hat{x}_{p}, \\
& \hat{y}_{g}=\sqrt{L^{2}-\left(\hat{x}_{p}\right)^{2}} .
\end{aligned}
$$

The goal point coordinates for $\left|\hat{x}_{p}\right|>L$ are computed from (9).

Finally, the transformation to the local frame yields

$$
x_{g}=\left(\cos \left(\theta^{c}\right) \sin \left(\theta^{c}\right)\right)\left[\begin{array}{l}
\hat{x}_{g} \\
\hat{y}_{g}
\end{array}\right] \text {, }
$$

which can be used with (4) to provide the curvature set-point at instant $k T_{o}$. A constant speed set-point can be specified depending on the corridor width.

4.3. Person Following. The objective is to pursue a person walking ahead of the vehicle in a natural way [18]. For this purpose, the goal point is chosen as a laser point belonging to the person. Due to this reactive solution, the distance $L$ is not constant, but is given by the selected range at each control step.

Initially, the target is locked by defining a rendezvous point $\mathbf{q}_{0}^{*}$ at a constant distance $d_{s}$ in the local $Y$ axis. In this way, the vehicle only starts moving when a person approaches $\mathbf{q}_{0}^{*}$.

Then, given that the robot is actually following the person, it is assumed that his/her relative position will be similar in consecutive scans. This assumption is acceptable if the outer loop period $T_{o}$ is small enough. Thus, laser data processing requires finding the point $\mathbf{q}_{k}(j)$ that is the closest to the previous goal $\mathbf{q}_{k-1}^{*}$. This is used to update the position of the goal point.

The closeness of the current laser points $\mathbf{q}_{k}(j)$ to the previous goal point is provided by the euclidean distance:

$$
d_{k}(j)=\sqrt{\left(x_{k}(j)-x_{k-1}^{*}\right)^{2}+\left(y_{k}(j)-y_{k-1}^{*}\right)^{2}},
$$

where the local cartesian coordinates are obtained with (6). if

Then, a laser reading $\mathbf{q}_{k}(i)$ is chosen as the goal point $\mathbf{q}_{k}^{*}$

$$
d_{k}(i)=\min _{j=0 \cdots N}\left(d_{k}(j)\right)
$$

and the distance $d_{k}(i)$ to the previous goal point is less than a certain threshold $d_{\text {th }}$ (see Figure 7). 


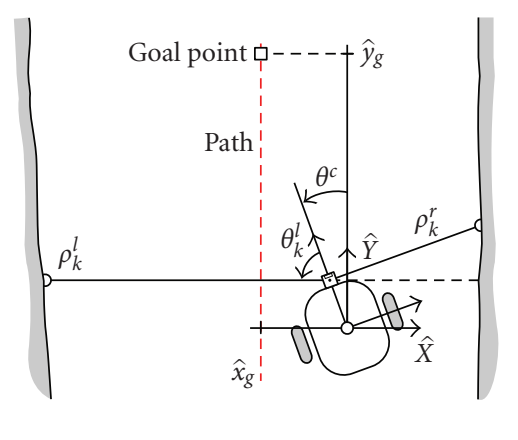

(a)

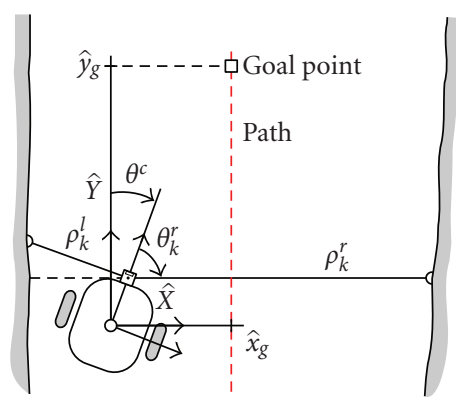

(b)

FIGURE 6: Corridor following using a $180^{\circ}$ partial field of view scanner, when (a) $\theta_{k}^{r}=-90^{\circ}$, or (b) $\theta_{k}^{l}=90^{\circ}$.

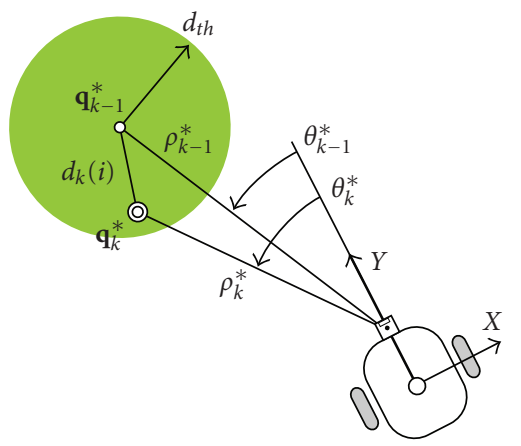

FIgURE 7: Selection of the goal point for person following.

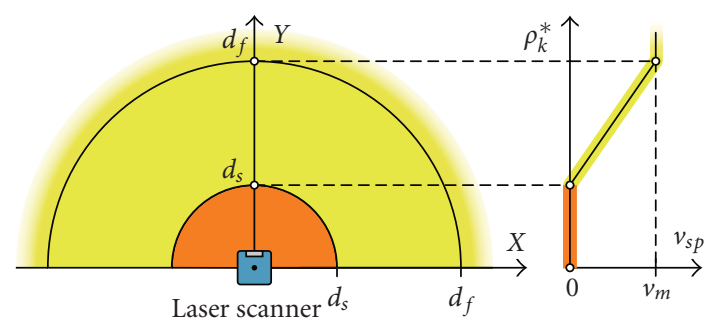

Figure 8: Relation between target distance and speed profile for person following.

If no valid current goal point can be found, the vehicle is stopped in standby for a new rendezvous and $\mathbf{q}_{k}^{*}$ is reinitialized as $\mathbf{q}_{0}^{*}$. Otherwise, the curvature set-point is obtained by applying (4) with

$$
\begin{aligned}
& x_{g}=-\rho_{k}^{*} \sin \left(\theta_{k}^{*}\right), \\
& L^{2}=\rho_{k}^{*}\left(2 d_{y} \cos \left(\theta_{k}^{*}\right)+\rho_{k}^{*}\right) .
\end{aligned}
$$

Regarding the speed set-point of the vehicle, this is computed according to the distance $\rho_{k}^{*}$ between the laser scanner and the goal point (see Figure 8):

$$
v_{s p}= \begin{cases}0, & \text { if } \rho_{k}^{*}<d_{s}, \\ \frac{\rho_{k}^{*}-d_{s}}{d_{f}-d_{s}} v_{m}, & \text { if } d_{s} \leq \rho_{k}^{*} \leq d_{f}, \\ v_{m}, & \text { if } \rho_{k}^{*}>d_{f},\end{cases}
$$

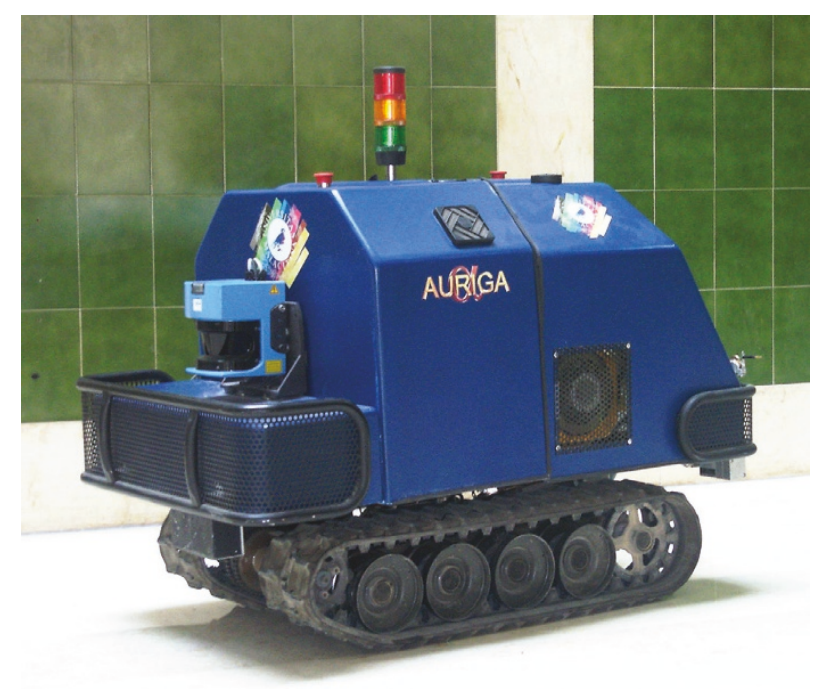

Figure 9: The tracked mobile robot Auriga- $\alpha$.

which is proportional within the limits defined by $d_{s}$ and $d_{f}$. In this way, $d_{s}$ represents the distance where the mobile robot begins its movement, which coincides with the rendezvous distance. The maximum speed $v_{m}$ is used whenever the goal point moves farther than $d_{f}$.

\section{Experimental Results}

The autonomous tracked vehicle Auriga- $\alpha$ has been employed for the experiments that illustrate the paper (see Figure 9). The mobile robot weights $260 \mathrm{~kg}$, and it is powered by an onboard petrol-fed AC generator of $4 \mathrm{~kW}$. The locomotion system is based on the skid-steering principle. Two DC motors with encoders independently move the rubber tracks. The maximum speed of the vehicle $v_{m}=1 \mathrm{~m} / \mathrm{s}$ can only be reached in straight line motion and decreases to zero according to the increase in the demanded curvature.

A Sick LMS 200 time-of-flight laser scanner is mounted on the forward part of the vehicle, at a distance of $d_{y}=0.5 \mathrm{~m}$ ahead of its coordinate center and $0.55 \mathrm{~m}$ above the ground. The scan plane is parallel to the motion plane, with the sensor $Y$ axis aligned with the forward motion direction. The 


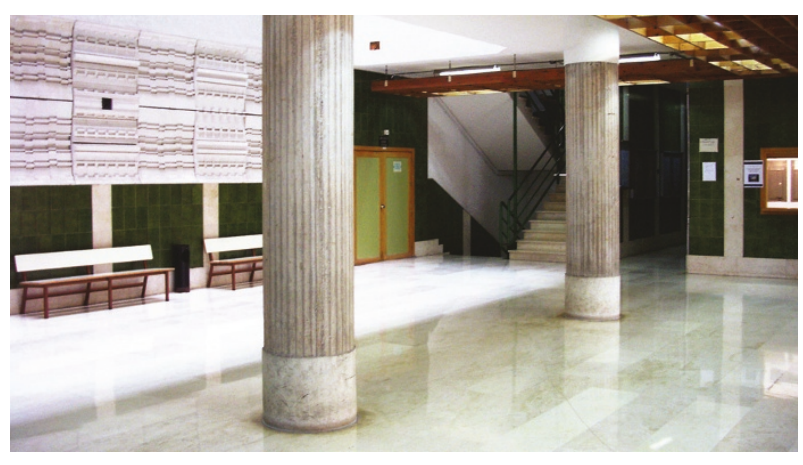

Figure 10: Experimental site.

main features of this laser device are the following: maximum range of $81.9 \mathrm{~m}, \Theta=180^{\circ}$ partial field of view, angular resolution $\Delta \theta=0.5^{\circ}$ (i.e., $N=360$ ), and range resolution of $1 \mathrm{~cm}$. A complete new scan takes $270 \mathrm{~ms}$ to be transmitted to the onboard computer, although it is acquired in just 27 ms. According to the manufacturer's specifications, the range errors are $\pm 4 \mathrm{~cm}$ for an exploration horizon of $20 \mathrm{~m}$.

The pure-pursuit path tracker is executed by an industrial computer, based on a Pentium-IV microprocessor at 2.2 GHz, via a real-time operating system (LynxOS 4.0). Pure-pursuit curvature set-points are computed from laser range data every $T_{o}=270 \mathrm{~ms}$. Odometric data are updated every $T_{i}=30 \mathrm{~ms}$. An approximate kinematic model of Auriga- $\alpha$ [34] has been employed for dead reckoning and to translate curvature and speed set-points into track speed references.

Several experiments have been carried out in an indoor environment, the hall of a university building, with several glass doors, benches, columns, stairs, and corridors (see Figure 10). Odometric data along with laser scans were recorded in order to obtain accurate motion estimations for performance evaluation. This was addressed offline using a hybrid GA-ICP laser scan matching technique [20]. By incrementally replaying motion estimations with recorded laser points, it is possible to map the environment and plot the actual path followed by Auriga- $\alpha$. For simplicity, the global frame in each experiment coincides with the initial pose of the vehicle.

The reactive path tracking strategies presented above have been implemented within a navigation architecture, where a global mission can be defined as a combination of different types of implicit and explicit paths. In these experiments, arbitration is simply performed from a teleoperator station that issues a signal to change the path type, that is, the goal point selection strategy for pure-pursuit.

Besides, a navigation safety module issues a motion stop signal if any laser-scan range falls below a threshold distance of $0.5 \mathrm{~m}$. In this way, if the obstacle eventually moves away, the mobile robot can resume its trajectory. This is a conservative strategy due to the relatively big size of the vehicle for indoors.

Two representative experiments composed of different types of path segments are described below. The first one

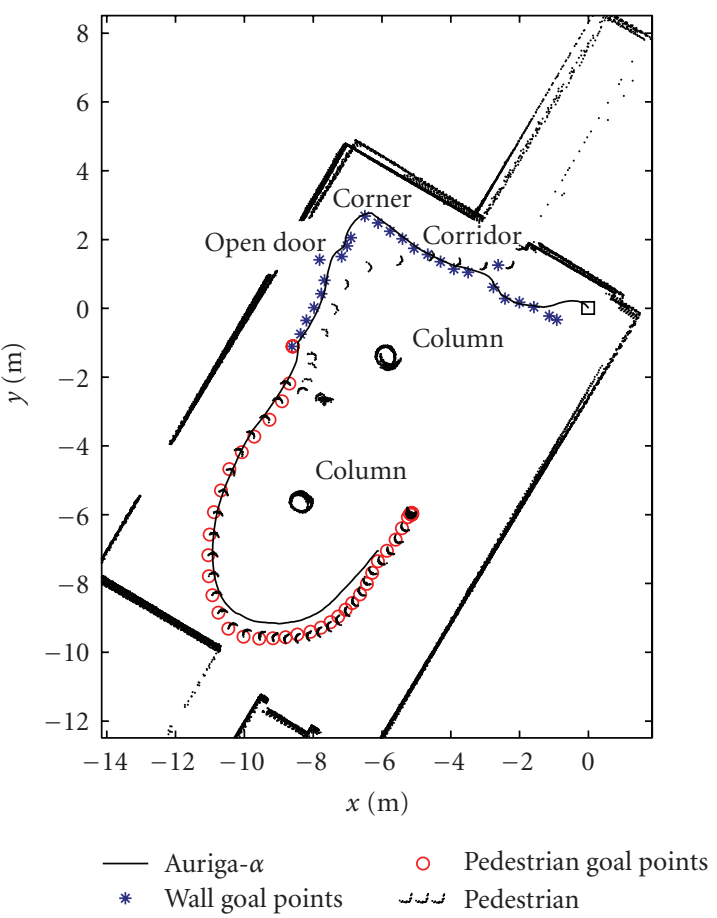

FIGURE 11: Tracked path along an indoor hall while following a wall and a person.

consists of two implicit path types. The second experiment combines an explicit path with an implicit path.

5.1. Following a Wall and a Person. In the first experiment, the Auriga- $\alpha$ robot has to track a right wall up to a rendezvous point, where it has to wait for a person to be followed. The pure-pursuit parameters are $v_{s p}=0.5 \mathrm{~m} / \mathrm{s}, L=$ $0.9 \mathrm{~m}$, and $d_{w}=1.5 \mathrm{~m}$ for wall following, and $d_{\mathrm{th}}=0.54 \mathrm{~m}$, $d_{s}=0.8 \mathrm{~m}$, and $d_{f}=1.5 \mathrm{~m}$ for person following.

The resulting path is shown in Figure 11. For the sake of clarity, the figure only represents one of every three consecutive scans, that is, $0.81 \mathrm{~s}$ intervals. A square denotes the initial position of the vehicle, and goal points are shown with asterisks and circles for the first and second segments of the experiment, respectively. The actual speed and curvature are shown in Figure 12.

The initial pose of the robot is too close to the wall, so in the beginning a high curvature set-point is produced by the control law. Precisely, this proximity causes that, momentarily, the safety module issues a null speed set-point just after the beginning.

Wall following overcomes an inner corner as well as two relevant gaps that correspond to a corridor entrance and an open door. If the gaps were wider, the wall-following tracking would turn into them. In fact, at about $t=6 \mathrm{~s}$ and $t=17 \mathrm{~s}$ path estimation and goal points are momentarily generated in this sense. If the mission goal was to enter through these gaps, the arbitrator would have to trigger a new path tracking type (e.g., corridor following).

When person following is activated, the vehicle stops waiting for the rendezvous. After a few seconds, it begins to 


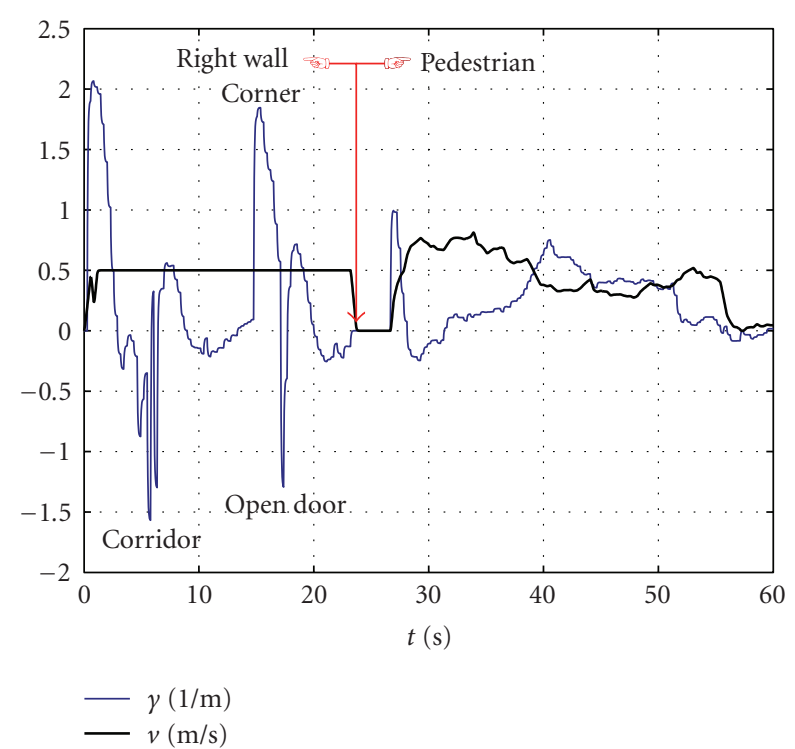

Figure 12: Curvature and speed of Auriga- $\alpha$ when following a wall and a person.

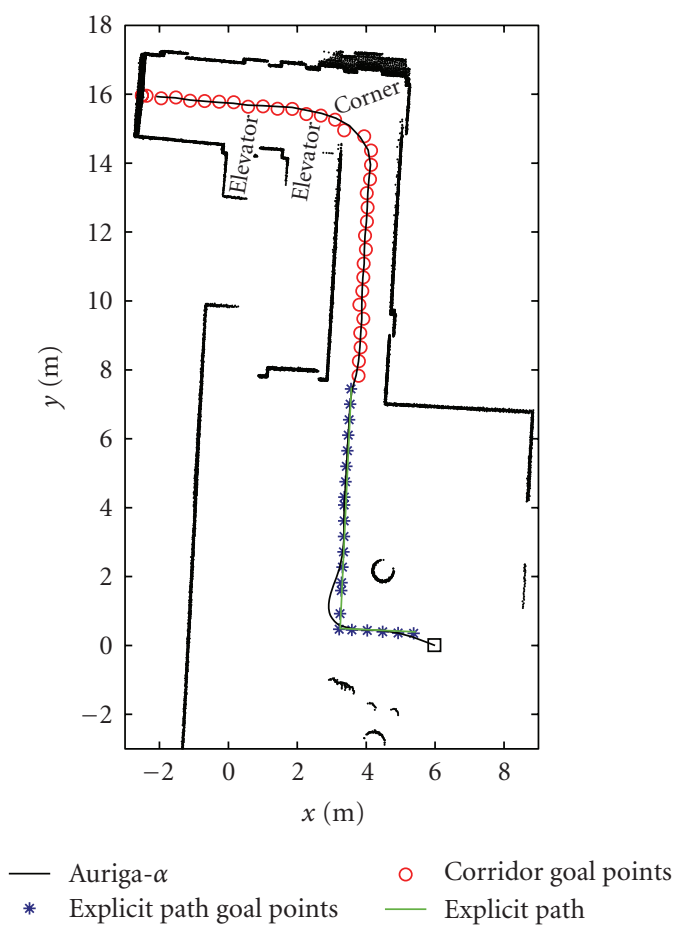

FIGURE 13: Tracked path along an indoor hall while following an explicit path and a corridor.

follow the person. It can be observed that the vehicle's speed is not constant but is adapted to that of the target. The goal point is chosen from the laser readings of the person so that the relative pose is similar to that of the previous control interval. This is why the goal points shown in Figure 11 are not necessarily the closest to the robot. Note that since the tracker strategy is to pursue the person, the robot path does not exactly coincide with the person's path.

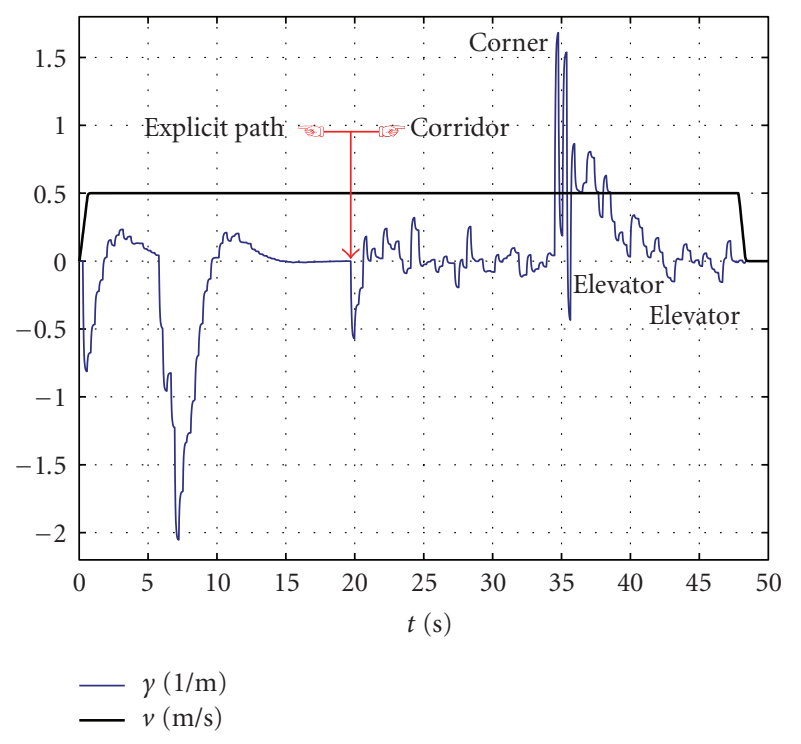

Figure 14: Curvature and speed of Auriga- $\alpha$ when following an explicit path and a corridor.

5.2. Following an Explicit Path and a Corridor. In this experiment, an explicit path takes the vehicle to the entrance of a corridor that has to be traversed. The actual path and the corresponding goal points are shown in Figure 13. The goal points from the precomputed geometric path are represented as asterisks, and those for the implicit corridor centerline as circles. The lookahead distance $L=0.9 \mathrm{~m}$ and the speed set-point $v_{s p}=0.5 \mathrm{~m} / \mathrm{s}$ are the same in both cases, and $\Delta \theta_{\text {th }}=45^{\circ}$ for corridor following.

The actual curvature and speed of Auriga- $\alpha$ for this experiment are represented in Figure 14. Note that the speed is constant during the transition between both path types. In this moment, only the goal point selection criterion is changed in the pure-pursuit controller.

To follow the explicit path, global pose estimation has been addressed by GA-ICP scan matching. At the beginning, the mobile robot reaches the explicit path in a smooth way due to the lookahead distance. Note that tracking errors appear during the abrupt transition between the straight line segments that compose the explicit path. When the end of the explicit path is reached, the experiment continues in a $L$-shaped corridor $1.8 \mathrm{~m}$ wide that becomes $2.5 \mathrm{~m}$ wide after the corner. Corridor disturbances such as the corner and the open doors of the elevators are overcome by the tracker.

5.3. Parameter Tuning. This section analyzes the effect of varying one particular design parameter for the same experimental setups presented above. Two performance indexes have been considered. One is related to the control effort, and the other to the tracking error. Both have been computed offline based on recorded data. The control effort is scored as the average absolute value of the curvature increments for all the control intervals. The tracking error is calculated as the average distance between the center of the robot and the closest point of the reference path. 
TABLE 1: Influence of the lookahead distance for wall following.

\begin{tabular}{lccccc}
\hline$L(\mathrm{~m})$ & 0.3 & 0.6 & 0.9 & 1.2 & 1.5 \\
\hline Control effort $(1 / \mathrm{m})$ & 0.2992 & 0.0514 & 0.0284 & 0.0175 & 0.0097 \\
Tracking error $(\mathrm{m})$ & 0.0563 & 0.1001 & 0.1731 & 0.2515 & 0.3390 \\
\hline
\end{tabular}

TABLE 2: Influence of speed for corridor following.

\begin{tabular}{lccccc}
\hline$v(\mathrm{~m} / \mathrm{s})$ & 0.1 & 0.3 & 0.5 & 0.7 & 0.9 \\
\hline Control effort $(1 / \mathrm{m})$ & 0.0079 & 0.0113 & 0.0172 & 0.0223 & 0.0238 \\
Tracking error $(\mathrm{m})$ & 0.1021 & 0.1102 & 0.1238 & 0.1498 & 0.1646 \\
\hline
\end{tabular}

TABLE 3: Influence of $d_{f}$ for person following.

\begin{tabular}{lccccc}
\hline$d_{f}(\mathrm{~m})$ & 1 & 1.25 & 1.5 & 1.75 & 2 \\
\hline Control effort $(1 / \mathrm{m})$ & 0.0121 & 0.0106 & 0.0107 & 0.0111 & 0.0117 \\
Tracking error $(\mathrm{m})$ & 0.2738 & 0.3492 & 0.4202 & 0.4919 & 0.5742 \\
\hline
\end{tabular}

Table 1 shows the effect of changing the lookahead distance for the $L$-shaped wall-following segment of the experiment described in Section 5.1. As the lookahead distance increases, the control effort diminishes whereas the tracking error becomes greater. Note that with $L=0.3 \mathrm{~m}$, the control effort raises considerably, which was evident as a shaky motion of the vehicle.

The distance of the implicit path to the wall $d_{w}$ has also been varied keeping $v_{s p}=0.5 \mathrm{~m} / \mathrm{s}$ and $L=0.9 \mathrm{~m}$. In this case, only when $d_{w}<1.1 \mathrm{~m}$, the mobile robot cannot turn the inner corner because it is too close to the wall and the safety stopping behavior activates. Since the vehicle's width is $0.75 \mathrm{~m}$, the gap between the robot and the wall is only $0.725 \mathrm{~m}$ for $d_{w}=1.1 \mathrm{~m}$.

Results from applying different speeds to the corridorfollowing experiment presented in Section 5.2 are summarized in Table 2. It can be observed that increasing speed raises both control effort and tracking error in a linear way. In addition, the threshold $\Delta \theta_{\text {th }}$ to select points from the left and right walls has been varied. Only when $\Delta \theta_{\mathrm{th}}>61^{\circ}$, the vehicle stops for security reasons during the turning of the inner corner because the goal points are not properly selected.

For the person-following experiment, the speed profile shown in Figure 8 has been modified by changing the target distance limit $d_{f}$ that corresponds to the maximum speed $v_{m}$. Results in Table 3 indicate that control effort is not affected substantially by this parameter. However, tracking error, which in this case is related to the closest point of the actual person path, increases with $d_{f}$. Note that tracking errors are considerably larger than those of the previous tables. This is because the lookahead $L$ is always greater than $\sqrt{d_{y}^{2}+d_{s}^{2}}$, so, with $d_{s}=0.8 \mathrm{~m}$ and $d_{y}=0.5 \mathrm{~m}, L>0.943 \mathrm{~m}$.

The distance to the previous goal point $d_{\text {th }}$ has been tested for person following under the conditions described in Section 5.1. Good results have been obtained with $d_{\text {th }}>$ $0.45 \mathrm{~m}$. Otherwise, the person is frequently lost.

\section{Conclusions}

The paper proposes the use of the pure-pursuit method for reactive path tracking of autonomous nonholonomic vehicles equipped with a $2 \mathrm{D}$ laser scanner. In this way, reactive navigation can profit from the efficiency of purepursuit with only a reduced number of easily adjustable design parameters.

To follow different path types, only the selection of the goal point requires a different treatment. This implies that robotic missions can be specified as a sequence of implicit and/or explicit paths that can be tracked with the same control law. Thus, our method naturally integrates motion control strategies that are commonly addressed separately, such as landmark-based navigation, person following, and geometric navigation.

An efficient range data interpretation has been developed for goal point selection on implicit paths. Only the essential information for a particular path type is extracted by considering the complete $2 \mathrm{D}$ scan reading.

Several experiments have been carried out in an indoor environment with the tracked mobile robot Auriga- $\alpha$. The proposed controller has followed paths in a reliable and smooth way with real-world features. Moreover, the influence of design parameters has also been studied experimentally.

For future work, the integration of pure-pursuit with reactive potential-field navigation can be useful for vehicles with nonholonomic constraints. It is also interesting to implement a sensor-based context-dependent arbitrator for switching between different path types as well as automatic tuning of path tracking parameters such as speed or lookahead.

\section{Acknowledgment}

This work was partially supported by the Spanish Project DPI2008-00533.

\section{References}

[1] A. Ollero and O. Amidi, "Predictive path tracking of mobile robots. Application to the CMU NavLab," in Proceedings of the 5th International Conference on Advanced Robotics (ICAR'91), vol. 2, pp. 1081-1086, Pisa, Italy, June 1991.

[2] A. J. García-Cerezo, A. Ollero, and J. L. Martínez, "Design of a robust high-performance fuzzy path tracker for autonomous vehicles," International Journal of Systems Science, vol. 27, no. 8, pp. 799-806, 1996.

[3] O. Amidi, "Integrated mobile robot control," Tech. Rep. CMURI-TR-90-17, Robotics Institute, Carnegie Mellon University, Pittsburgh, Pa, USA, May 1990.

[4] B. M. Leedy, J. S. Putney, C. Bauman, S. Cacciola, J. M. Webster, and C. F. Reinholtz, "Virginia Tech's twin contenders: a comparative study of reactive and deliberative navigation," Journal of Field Robotics, vol. 23, no. 9, pp. 709-727, 2006.

[5] N. Montés, M. C. Mora, and J. Tornero, "Trajectory generation based on rational bezier curves as clothoids," in Proceedings of IEEE Intelligent Vehicles Symposium (IV '07), pp. 505-510, Istanbul, Turkey, June 2007. 
[6] K. O. Arras, N. Tomatis, B. T. Jensen, and R. Siegwart, "Multisensor on-the-fly localization: precision and reliability for applications," Robotics and Autonomous Systems, vol. 34, no. 2-3, pp. 131-143, 2001.

[7] R. Wallace, A. Stentz, C. E. Thorpe, H. Moravec, W. Whittaker, and T. Kanade, "First results in robot road-following," in Proceedings of the 9th International Joint Conference on Artificial Intelligence (IJCAI '85), vol. 1, pp. 66-71, Los Angeles, Calif, USA, August 1985.

[8] J. L. Martínez, A. Pozo-Ruz, S. Pedraza, and R. Fernández, "Object following and obstacle avoidance using a laser scanner in the outdoor mobile robot Auriga- $\alpha$," in Proceedings of IEEE/RSJ International Conference on Intelligent Robots and Systems (IROS '98), vol. 1, pp. 204-209, Victoria, Canada, October 1998.

[9] R. C. Arkin, Behavior-Based Robotics, The MIT Press, Cambridge, Mass, USA, 1998.

[10] M. A. Martínez and J. L. Martínez, "The dual-frequency sonar system of the mobile robot RAM-2," Robotica, vol. 22, no. 3, pp. 263-270, 2004.

[11] E. Aguirre and A. González, "Integrating fuzzy topological maps and fuzzy geometric maps for behavior-based robots," International Journal of Intelligent Systems, vol. 17, no. 3, pp. 333-368, 2002.

[12] A. Mandow, J. M. Gómez-de-Gabriel, J. L. Martínez, V. F. Muñoz, A. Ollero, and A. J. García-Cerezo, "The autonomous mobile robot AURORA for greenhouse operation," IEEE Robotics \& Automation Magazine, vol. 3, no. 4, pp. 18-28, 1996.

[13] D. Ratner and P. McKerrow, "Navigating an outdoor robot along continuous landmarks with ultrasonic sensing," Robotics and Autonomous Systems, vol. 45, no. 2, pp. 73-82, 2003.

[14] M. Harb, R. Abielmona, E. Petriu, and K. Naji, "Neural control system of a mobile robot," in Proceedings of IEEE International Joint Conference on Neural Networks (IJCNN '08), pp. 28252832, Hong Kong, June 2008.

[15] O. C. Barawid Jr., A. Mizushima, K. Ishii, and N. Noguchi, "Development of an autonomous navigation system using a two-dimensional laser scanner in an orchard application," Biosystems Engineering, vol. 96, no. 2, pp. 139-149, 2007.

[16] J. H. Lee, K. Abe, T. Tsubouchi, R. Ichinose, Y. Hosoda, and K. Ohba, "Collision-free navigation based on people tracking algorithm with biped walking model," in Proceedings of IEEE/RSJ International Conference on Intelligent Robots and Systems (IROS '08), pp. 2983-2989, Nice, France, September 2008.

[17] T. Yoshimi, M. Nishiyama, T. Sonoura, et al., "Development of a person following robot with vision based target detection," in Proceedings of IEEE/RSJ International Conference on Intelligent Robots and Systems (IROS '06), pp. 5286-5291, Beijing, China, October 2006.

[18] R. Gockley, J. Forlizzi, and R. Simmons, "Natural personfollowing behavior for social robots," in Proceedings of the ACM/IEEE Conference on Human-Robot Interaction (HRI '07), pp. 17-24, Arlington, Va, USA, March 2007.

[19] T.-H. S. Li, S.-J. Chang, and W. Tong, "Fuzzy target tracking control of autonomous mobile robots by using infrared sensors," IEEE Transactions on Fuzzy Systems, vol. 12, no. 4, pp. 491-501, 2004.

[20] J. L. Martínez, J. González, J. Morales, A. Mandow, and A. J. García-Cerezo, "Mobile robot motion estimation by 2D scan matching with genetic and iterative closest point algorithms," Journal of Field Robotics, vol. 23, no. 1, pp. 21-34, 2006.
[21] L. L. Scharf, W. P. Harthill, and P. H. Moose, "A comparison of expected flight times for intercept and pure-pursuit missiles," IEEE Transactions on Aerospace and Electronic Systems, vol. 5, no. 4, pp. 672-673, 1969.

[22] R. C. Coulter, "Implementation of the pure-pursuit path tracking algorithm,” Tech. Rep. CMU-RI-TR-92-01, Robotics Institute, Carnegie Mellon University, Pittsburgh, Pa, USA, 1992.

[23] A. Ollero, A. J. García-Cerezo, and J. L. Martínez, "Fuzzy supervisory path tracking of mobile robots," Control Engineering Practice, vol. 2, no. 2, pp. 313-319, 1994.

[24] K. N. Murphy, "Analysis of robotic vehicle steering and controller delay," in Proceedings of the 5th International Symposium on Robotics and Manufacturing (ISRAM '94), pp. 1-6, Maui, Hawaii, USA, August 1994.

[25] A. Ollero and G. Heredia, "Stability analysis of mobile robot path tracking," in Proceedings of IEEE/RSJ International Conference on Intelligent Robots and Systems (IROS '95), vol. 3, pp. 461-466, Pittsburgh, Pa, USA, August 1995.

[26] A. Rodríguez-Castaño, G. Heredia, and A. Ollero, "Analysis of a GPS-based fuzzy supervised path tracking system for large unmanned vehicles," in Proceedings of the 4th IFAC International Symposium on Intelligent Components and Instruments for Control Applications (SICICA '00), pp. 141-146, Buenos Aires, Argentina, September 2000.

[27] J. L. Martínez, J. Morales, A. Mandow, and A. J. García-Cerezo, "Steering limitations for a vehicle pulling passive trailers," IEEE Transactions on Control Systems Technology, vol. 16, no. 4, pp. 809-818, 2008.

[28] T. Hellström, T. Johansson, and O. Ringdahl, "Development of an autonomous forest machine for path tracking," in Field and Service Robotics, chapter 50, pp. 603-614, Springer, New York, NY, USA, 2006.

[29] K. Petrinec, Z. Kovačić, and A. Marozin, "Simulator of multiAGV robotic industrial environments," in Proceedings of IEEE International Conference on Industrial Technology (ICIT '03), vol. 2, pp. 979-983, Maribor, Slovenia, December 2003.

[30] C. Urmson, C. Ragusa, D. Ray, et al., "A robust approach to high-speed navigation for unrehearsed desert terrain," Journal of Field Robotics, vol. 23, no. 8, pp. 467-508, 2006.

[31] A. Stentz, C. Dima, C. Wellington, H. Herman, and D. Stager, "A system for semi-autonomous tractor operations," Autonomous Robots, vol. 13, no. 1, pp. 87-104, 2002.

[32] J. Wit, C. D. Crane III, and D. Armstrong, "Autonomous ground vehicle path tracking," Journal of Robotic Systems, vol. 21, no. 8, pp. 439-449, 2004.

[33] A. Pozo-Ruz, J. L. Martínez, and A. J. García-Cerezo, "Integration of a rotary sonar in the mobile robot RAM-2," in Proceedings of the 3rd IFAC International Symposium on Intelligent Components and Instruments for Control Applications (SICICA '97), pp. 147-151, Annecy, France, June 1997.

[34] J. L. Martínez, A. Mandow, J. Morales, S. Pedraza, and A. J. García-Cerezo, "Approximating kinematics for tracked mobile robots," The International Journal of Robotics Research, vol. 24, no. 10, pp. 867-878, 2005. 